\title{
Canonical Noether symmetries and commutativity properties for gauge systems
}

\author{
Xavier Gràcia ${ }^{\mathrm{a})}$ \\ Departament de Matemàtica Aplicada i Telemàtica, Universitat Politècnica de Catalunya, \\ Campus Nord UPC, edifici C3, C. Jordi Girona 1, \\ 08034 Barcelona, Catalonia, Spain \\ Josep M. Pons ${ }^{\text {b) }}$ \\ Departament d'Estructura $i$ Constituents de la Matèria, \\ Universitat de Barcelona and Institut de Física d'Altes Energies, Av. Diagonal 647, \\ 08028 Barcelona, Catalonia, Spain
}

(Received 3 February 2000; accepted for publication 20 June 2000)

For a dynamical system defined by a singular Lagrangian, canonical Noether symmetries are characterized in terms of their commutation relations with the evolution operators of Lagrangian and Hamiltonian formalisms. Separate characterizations are given in phase space, in velocity space, and through an evolution operator that links both spaces. (C) 2000 American Institute of Physics.

[S0022-2488(00)01710-2]

\section{INTRODUCTION}

Most physical theories implement the dynamics as a result of the application of a variational principle, that is, by means of a Lagrangian. Among the dynamical symmetries of these theories, that is, transformations that map solutions of the equations of motion into solutions, we can single out the Noether symmetries, that is, the continuous transformations that leave the action invariant-except for boundary terms. In addition, if we aim to move the description of the dynamics from the tangent bundle (velocity space) TQ of its configuration space $Q$ to the cotangent bundle (phase space) $\mathrm{T}^{*} Q$, other distinctions can be raised as to whether the symmetry transformation in velocity space is projectable to phase space and, in the affirmative case, whether the transformation in phase space is canonical. We will consider time-independent Lagrangians, as it is the usual case in physical theories, but we will allow to deal with time-dependent functions to cover also gauge symmetries (symmetries depending upon arbitrary functions of time, or spacetime variables in field theory); then we will use $\mathbf{R} \times \mathrm{T} Q$ and $\mathbf{R} \times \mathrm{T}^{*} Q$ instead of $\mathrm{T} Q$ and $\mathrm{T}^{*} Q$.

The infinitesimal symmetries of an ordinary dynamical system are characterized by a property of commutativity: essentially, that the time evolution operator commutes with the operator that generates the symmetry. Let us state with more detail this result, which is standard for theories with no gauge freedom, using differential-geometric language. Let $\mathbf{X}$ be the vector field that governs the dynamics (the time evolution) of some system on a given manifold $\mathcal{M}$ ( $\mathcal{M}$ can be, for instance, $\mathbf{R} \times \mathrm{T} Q$ or $\mathbf{R} \times \mathrm{T}^{*} Q$ for some configuration manifold $Q ; \mathbf{R}$ parametrizes the independent variable-the time). For an open interval $I \subset \mathbf{R}$, a path $\gamma: I \rightarrow \mathcal{M}$ is a solution to the dynamics if $\dot{\gamma}=\mathbf{X} \circ \gamma$. Let a vector field $\mathbf{V}$ be a candidate for a symmetry of the dynamics defined by $X$. Then the flow of $\mathbf{V}$ (a local one-parameter group of diffeomorphisms) transforms solutions into solutions if and only if $\mathbf{X}$ is $\mathbf{V}$-invariant, that is to say,

$$
\mathcal{L}_{\mathbf{V}} \mathbf{X}=[\mathbf{V}, \mathbf{X}]=0,
$$

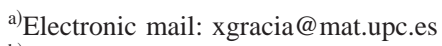

${ }^{b)}$ Electronic mail: pons@ecm.ub.es 
where $\mathcal{L}_{\mathbf{V}}$ stands for the Lie derivative. This is an immediate consequence of the well-known fact that $[\mathbf{V}, \mathbf{X}]=0$ iff their flows commute. ${ }^{1-3}$

Our aim in this paper is to obtain some generalized versions of this result. More precisely, our purpose is to study how the canonical Noether transformations implement this commutativity requirement in the general case of gauge theories (those derived from singular Lagrangians). Instead of providing with new procedures to determine symmetries, we give alternative ways to characterize them, associated with a specific property of commutativity. Recall that the variation of the Lagrangian under a Noether symmetry is a total derivative; this statement is far from expressing any kind of commutativity. We will discover however that one can characterize canonical Noether symmetries through commutativity properties; in this way, we give a new perspective, with a geometrical flavor, to identify the Noether symmetries of a dynamical system. This approach can be applied in particular to gauge theories, where it can be used as a direct test as to whether a given transformation is a Noether symmetry.

Since many dynamical systems - and, among them, those describing the fundamental interactions - have room for gauge freedom, we will assume in our framework that the Lagrangian may be singular. To be more concrete, we will consider theories described by time-independent first-order Lagrangians whose Hessian matrix with respect to the velocities may be singular. In this case the conversion from tangent space language to phase space language has some peculiarities: there are constraints in the formalism, the dynamics has some degree of arbitrariness, etc. This is nothing but the framework first studied by Dirac to deal with gauge theories or, more generally, constrained systems. ${ }^{4-9}$ The regular case is recovered when no Hamiltonian constraints occur.

Throughout the paper we will only consider continuous symmetries. Among them, how can we distinguish the Noether symmetries? The distinction comes in part from the following fact: a Noether symmetry has an associated conserved quantity, and this conserved quantity contains all the information to reconstruct the symmetry. ${ }^{1}$ This fact characterizes a Noether symmetry for regular Lagrangians (those with regular Hessian matrix), but not in the general case of gauge theories that we are also addressing: there are symmetries with conserved quantities that are not Noether.

Let us distinguish clearly the singular case from the regular one. In the regular case we know that:

(i) there is a one-to-one correspondence between Noether symmetries and conserved quantities;

(ii) when formulated in phase space, the conserved quantities become the generators, through the Poisson bracket, of the Noether symmetries, therefore, Noether symmetries are canonical transformations.

Instead, in the case including gauge theories, we can list a very different set of assertions:

(a) There can be conserved quantities in phase space that do not generate symmetries at all.

(b) There can be conserved quantities in phase space that generate symmetries that are not Noether.

(c) There can be nontrivial Noether symmetries whose conserved quantity in velocity space is identically vanishing.

(d) There can be Noether transformations in tangent space that are not projectable to phase space (but the conserved quantity is always projectable).

(e) It remains true that, regardless as to whether the Noether symmetry is projectable or not to phase space, it can be always reconstructed through the Poisson bracket by using the conserved quantity in phase space. In other words, the conserved quantity still encodes all the information to reconstruct the symmetry.

(f) When the Noether symmetry is projectable to phase space, it is also true that such symmetry is always a canonical transformation that is generated by a conserved quantity. We call such a symmetry a canonical Noether transformation. 
Let us briefly comment on these assertions.

To prove (a) it suffices to realize that any second class constraint is a conserved quantity that does not generate a symmetry: it takes the motions out of the constraint surface.

Statement (b) is a consequence of the fact that the conserved quantities $G^{H}$ that generate canonical Noether transformations satisfy stricter conditions $\left[\mathbf{K} \cdot G^{H}=0\right.$, see Eq. (26) in Sec. III] than the ones required to generate dynamical symmetry transformations in phase space $\left(\mathbf{K} \cdot G^{H}\right.$ = quadratic constraints, see Ref. 10); this is illustrated at the end of the second example in Sec. IV.

The occurrence of (c) is studied in Ref. 11, and it happens when the number of independent primary Lagrangian constraints is less than the number of independent primary Hamiltonian constraints; the simplest example is given by the free relativistic particle, that does not have Lagrangian constraints.

An example of statement (d) is provided, in any time-independent gauge theory, by the Noether symmetry associated with time translations: the variation $\delta q=\dot{q}$ is not projectable to phase space, whereas its conserved quantity, the energy, projects to the Hamiltonian function. The projectability of the conserved quantity associated with any Noether transformation was noticed in Ref. 12. On the other hand, special situations may often arise when studying the projectability of the gauge transformations, as for example the nonexistence of Hamiltonian gauge generators of a certain model possessing Lagrangian gauge transformations, ${ }^{13}$ and the loss of covariance of the Hamiltonian gauge transformations for a particle model admitting a Lorentz covariant Hamiltonian formulation. ${ }^{14}$

Statement (e) is explained in Refs. 13 and 15, where several examples can be found. Finally, assertion (f) is proven in Ref. 16.

From these considerations, we see that it is important to characterize the conserved quantities, because they already encode the transformation. This is the usual procedure when one considers Noether symmetries. In this paper we propose a shift of emphasis: instead of focusing on the conserved quantities, we will be interested in properties of the transformations themselves. We will show the relevance of commutation properties in order to characterize Noether symmetries. In this sense, from a theoretical viewpoint we will enlarge the list of properties above; from a practical viewpoint we will provide with new instruments to check whether a given transformation is a Noether symmetry.

We organize the paper as follows. The basic notations and some preliminary results are set up in Sec. II. Section III is mainly devoted to the study of Noether transformations that are projectable to phase space; these transformations are given different characterizations in terms of commutation relations involving the evolution operators of the Hamiltonian and the Lagrangian formalisms. Section IV contains some examples illustrating these results, and Sec. V is devoted to conclusions.

\section{NOTATION AND PRELIMINARY RESULTS}

We consider a configuration space $Q$, with velocity space the tangent bundle $\mathrm{T} Q$, and a (time-independent, first-order) Lagrangian function $L(q, \dot{q})$ defined on it. The fiber derivative of $L$ defines the Legendre's transformation, which is a map from velocity space to phase space, $\mathcal{F} L: \mathrm{T} Q \rightarrow \mathrm{T}^{*} Q$, locally defined by

$$
\mathcal{F} L(q, \dot{q})=(q, \hat{p})
$$

where we have introduced the momenta $\hat{p}=\partial L / \partial \dot{q}$-we will suppress most indices.

Given a function $h(q, p)$ in phase space, its pull-back (through the Legendre's transformation $\mathcal{F} L)$ is the function $\mathcal{F} L^{*}(h)$ in velocity space obtained by substituting the momenta by their Lagrangian expression: $\mathcal{F} L^{*}(h)(q, \dot{q})=h(q, \hat{p})$. A function $f(q, \dot{q})$ in velocity space is called $\mathcal{F} L$-projectable—or, simply, projectable—if it is the pull-back of a certain function $h(q, p)$. 
We shall always assume that the Legendre's transformation $\mathcal{F} L$ has constant rank; this amounts to say that the fiber Hessian of $L$, which is locally described by the Hessian matrix with respect to the velocities

$$
W=\frac{\partial^{2} L}{\partial \dot{q} \partial \dot{q}},
$$

has constant rank. Notice that gauge symmetries can only exist when this rank is not maximal; this is the case we are interested in.

Let $\gamma_{\mu}\left(\mu=1, \ldots, p_{0}\right)$ be a basis of the null vectors of $W$; then the necessary and sufficient condition for a function $f(q, \dot{q})$ in $\mathrm{T} Q$ to be (locally) projectable to $\mathrm{T}^{*} Q$ is

$$
\Gamma_{\mu} \cdot f=0
$$

for each $\mu$, where the vector fields $\Gamma_{\mu}:=\gamma_{\mu}(\partial / \partial \dot{q})$ indeed span a basis of the kernel of the tangent map $\mathrm{T}(\mathcal{F} L)$.

Under the same assumption about the constant rank, the image $P_{0}$ of the Legendre's map can be locally taken as the submanifold of phase space described by the vanishing of $p_{0}$ primary Hamiltonian constraints $\phi_{\mu}$, linearly independent at each point of $P_{0}$. So they satisfy $\mathcal{F} L^{*}\left(\phi_{\mu}\right)=0$ by definition. Then the basis $\gamma_{\mu}$ can be taken as ${ }^{6}$

$$
\gamma_{\mu}:=\mathcal{F} L^{*}\left(\frac{\partial \phi_{\mu}}{\partial p}\right) .
$$

Though our Lagrangian is time-independent, we will need to consider time-dependent functions. The adjunction of the $t$-variable where needed will not cause any problem. The timederivative operator acting on a function $f(t, q, \dot{q})$ is

$$
\frac{\mathrm{d}}{\mathrm{d} t}=\frac{\partial}{\partial t}+\dot{q} \frac{\partial}{\partial q}+\ddot{q} \frac{\partial}{\partial \dot{q}},
$$

with the acceleration $\ddot{q}$ as an independent variable (this involves the tangent bundle of second order, $\mathrm{T}^{2} Q$ ). Then the Euler-Lagrange equations can be written

$$
[L]_{(q, \dot{q}, \ddot{q})}=0,
$$

where we have defined

$$
[L]:=\frac{\partial L}{\partial q}-\frac{\mathrm{d} \hat{p}}{\mathrm{~d} t}=\alpha-\ddot{q} W,
$$

with $\alpha=\partial L / \partial q-\dot{q}\left(\partial^{2} L / \partial q \partial \dot{q}\right)$. The primary Lagrangian constraints arise from it,

$$
\chi_{\mu}:=\alpha \gamma_{\mu}=[L] \gamma_{\mu},
$$

though they are not necessarily independent; their vanishing defines a subset $V_{1} \subset \mathrm{T} Q$.

As a matter of notation, it is usual to write $\underset{M}{f \approx 0}$ to mean that $f(x)=0$ for all $x \in M$ (Dirac's weak equality); for instance $\phi_{\mu} \underset{P_{0}}{\approx}$ and $\chi_{\mu} \approx 0$.

In a gauge theory the dynamics either in Lagrangian or Hamiltonian formalisms has a certain degree of arbitrariness. One can introduce a useful differential operator $\mathbf{K}$ connecting the Lagrangian and Hamiltonian formalisms, that has no ambiguity at all, and that still represents the dynamics. ${ }^{6}$ It can be defined as a vector field along the Legendre's transformation $\mathcal{F} L,{ }^{17}$ and, as a differential operator, it gives the time evolution of a function $h$ in $\mathbf{R} \times \mathrm{T}^{*} Q$ as a function $\mathbf{K} \cdot h$ in $\mathbf{R} \times \mathrm{T} Q$ by 


$$
\mathbf{K} \cdot h:=\mathcal{F} L *\left(\frac{\partial h}{\partial t}\right)+\mathcal{F} L *\left(\frac{\partial h}{\partial q}\right) \dot{q}+\mathcal{F} L^{*}\left(\frac{\partial h}{\partial p}\right) \frac{\partial L}{\partial q} .
$$

The operator $\mathbf{K}$ is directly determined by the Lagrangian by just taking partial derivatives. Instead, the determination of the dynamics either in tangent space or in phase space requires more involved computations. In this sense, $\mathbf{K}$ is the simplest among the evolution operators, and this will turn out to be advantageous in order to characterize the Noether symmetry transformations by way of commutativity properties. The operator $\mathbf{K}$ is especially valuable in the study of singular Lagrangians. For instance, all the Lagrangian constraints are obtained by applying it to the Hamiltonian constraints, ${ }^{18}$ and the Lagrangian and Hamiltonian dynamics can be described geometrically by using this operator. ${ }^{17}$ The operator $\mathbf{K}$ will be instrumental in obtaining some of the results of the next section.

It will prove very convenient to present two other equivalent expressions for the operator $\mathbf{K}$, to be used in the next section. The first one is

$$
\mathbf{K} \cdot h=\frac{\mathrm{d}}{\mathrm{d} t} \mathcal{F} L^{*}(h)+[L] \mathcal{F} L^{*}\left(\frac{\partial h}{\partial p}\right),
$$

whose proof is direct by using the chain rule. ${ }^{13}$ A direct consequence of this equation and definition (5) is another expression for the primary Lagrangian constraints:

$$
\chi_{\mu}=\mathbf{K} \cdot \phi_{\mu}
$$

The second expression relates $\mathbf{K}$ with the Hamiltonian evolution: ${ }^{6}$

$$
\mathbf{K} \cdot h=\mathcal{F} L^{*}\left(\frac{\partial h}{\partial t}\right)+\mathcal{F} L^{*}\{h, H\}+\sum_{\mu} \mathcal{F} L^{*}\left\{h, \phi_{\mu}\right\} v^{\mu} .
$$

Here $H$ is any Hamiltonian function (its pull-back to $\mathrm{T} Q$ is the Lagrangian energy; it is defined up to primary Hamiltonian constraints). And the $v^{\mu}(q, \dot{q})$ are functions uniquely determined by this equality when one takes $h=q^{i}$; these functions are not projectable, and indeed

$$
\boldsymbol{\Gamma}_{\nu} \cdot v^{\mu}=\delta_{\nu}^{\mu} .
$$

A consequence of (9) is a test of projectability for the function $\mathbf{K} \cdot h$ :

$$
\boldsymbol{\Gamma}_{\mu} \cdot(\mathbf{K} \cdot h)=\mathcal{F} L^{*}\left\{h, \phi_{\mu}\right\},
$$

so $\mathbf{K} \cdot h$ is projectable iff $h$ is a first-class function with respect to the primary Hamiltonian constraint submanifold $P_{0}$.

The Lagrangian time-evolution differential operator can be expressed ${ }^{6}$ as

$$
\mathbf{X}^{L}=\mathbf{X}_{0}^{L}+\eta^{\mu} \boldsymbol{\Gamma}_{\mu},
$$

where the $\eta^{\mu}$ are in principle arbitrary functions that express the gauge freedom of the timeevolution operator and $\mathbf{X}_{0}^{L}$ is a vector field in velocity space

$$
\mathbf{X}_{0}^{L}=\frac{\partial}{\partial t}+\dot{q}^{i} \frac{\partial}{\partial q^{i}}+a^{i}(q, \dot{q}) \frac{\partial}{\partial \dot{q}^{i}} .
$$

The accelerations $a^{i}$ in $\mathbf{X}_{0}^{L}$ may be determined by the formalism, with some arbitrariness owing to the gauge freedom, and we do not need here their explicit expression, which is given in. ${ }^{6}$ The nature of this operator has been recently discussed in Refs. 19, 20. In view of application we only need to know its relationship with the operator $\mathbf{K}:{ }^{18}$ 


$$
\mathbf{K} \cdot h=\mathbf{X}_{0}^{L} \cdot \mathcal{F} L^{*}(h)+\chi_{\mu} \frac{\partial v^{\mu}}{\partial \dot{q}} \mathcal{F} L^{*}\left(\frac{\partial h}{\partial p}\right) .
$$

\section{CANONICAL NOETHER TRANSFORMATIONS FOR GAUGE THEORIES}

Now we are ready to study the symmetries in Lagrangian and Hamiltonian formalisms as commutation relations between these symmetries and the dynamics. The case of gauge theories will lead to modified versions of Eq. (1) that account for the existence of constraints and the ambiguity of the dynamics due to gauge freedom.

Let us consider an infinitesimal Noether transformation $\delta^{L} q(t, q, \dot{q})$ in configuration space, that is to say, the variation of $L$ is a total time-derivative. Then a conserved quantity $G^{L}$ arises:

$$
[L]_{i} \delta^{L} q^{i}+\frac{\mathrm{d} G^{L}}{\mathrm{~d} t}=0 .
$$

As we have recalled in the introduction, the conserved quantity is always projectable ${ }^{12}$ to a function $G^{H}(t, q, p)$ in phase space, $G^{L}=\mathcal{F} L^{*}\left(G^{H}\right)$. This is proved by extracting the coefficient of the acceleration $\ddot{q}$ from Eq. (15) and then saturating the result with the null vectors $\gamma_{\mu}$ of the Hessian matrix $W$, thus obtaining $\boldsymbol{\Gamma}_{\mu} \cdot G^{L}=0$.

Notice that there is some arbitrariness in $G^{H}$ : nothing changes if we add to it a linear combination of the primary Hamiltonian constraints because $\mathcal{F} L^{*}\left(\phi_{\mu}\right)=0$ identically.

In this paper we will consider the case where the transformation itself is projectable to phase space, that is,

$$
\delta^{L} q=\mathcal{F} L^{*}\left(\delta^{H} q\right)
$$

for a certain $\delta^{H} q(t, q, p)$. Notice that there is also an arbitrariness in the determination of $\delta^{H} q$ because of the existence of Hamiltonian constraints.

Using $G^{H}$ and $\delta^{H}$, the Noether condition may be written

$$
[L]_{i} \mathcal{F} L^{*}\left(\delta^{H} q^{i}\right)+\frac{\mathrm{d} \mathcal{F} L^{*} G^{H}}{\mathrm{~d} t}=0
$$

from which, by extracting the coefficient of $\ddot{q}$, one obtains $W \mathcal{F} L^{*}\left(\delta^{H} q-\partial G^{H} / \partial p\right)=0$. From this equation, and using the null vectors of the Hessian, it is easy to redefine $G^{H}$ and $\delta^{H} q$ conveniently_using the primary Hamiltonian constraints-in order to obtain ${ }^{16}$

$$
\delta^{H} q^{i}=\frac{\partial G^{H}}{\partial p_{i}}=\left\{q^{i}, G^{H}\right\} .
$$

In other words: a projectable Noether transformation is canonically generated in phase space. On this basis we are ready to generalize Eq. (1) to the case of projectable Noether symmetries associated with singular Lagrangian dynamics. First we will give a characterization in phase space, next we will give a characterization using the operator $\mathbf{K}$, and finally we will give a characterization in velocity space.

\section{A. Characterization in phase space}

Now we wish to study the Noether transformations in phase space. The dynamics of gauge theories, as examples of constrained systems in the Dirac sense, exhibit a certain amount of arbitrariness in order to account for the gauge-unphysical—degrees of freedom. A typical evolution operator in phase space will be

$$
\mathbf{X}^{H} \approx \frac{\partial}{\partial t}+\{-, H\}+\lambda^{\mu}\left\{-, \phi_{\mu}\right\}
$$


where $\approx$ (Dirac's weak equality) is here an equality up to primary Hamiltonian constraints, and $\lambda^{\mu}$ are a set of arbitrary Lagrange multipliers. As a matter of fact, these Lagrange multipliers are determined as functions in tangent space just by applying (18) to the configuration variables, yielding $\lambda^{\mu}=v^{\mu}(q, \dot{q})$ —see (9).

Notice that the weak equality in (18) makes the definition of $\mathbf{X}^{H}$ consistent with any redefinition of the basis of primary constraints. However, this is not the final form of the dynamics. To get the final dynamics we must perform a stabilization algorithm: ${ }^{5-7,21,22}$ consistency requirements, that is, the tangency of $\mathbf{X}^{H}$ to the surface of constraints, may lead to new constraints and also to the determination of some of the Lagrangian multipliers as functions in phase space.

Notice that for any values we can give to the Lagrangian multipliers, the last piece in (18) may be written as $\{-$, phc $\}$, where phc stands for an arbitrary linear combination of the primary Hamiltonian constraints,

$$
\mathbf{X}^{H} \approx \frac{\partial}{\partial t}+\{-, H\}+\{-, \text { phc }\} .
$$

Let us consider the infinitesimal transformation generated by a vector field $\mathbf{V}^{H}$ in $\mathrm{T}^{*} Q$, that is to say, $\delta^{H} h=\mathbf{V}^{H} \cdot h$-an infinitesimal parameter may be understood here. The condition that $\mathbf{V}^{H}$ be a symmetry of the dynamics is no longer characterized by the strong condition of commutativity $\left[\mathbf{V}^{H}, \mathbf{X}^{H}\right]=0$. We may venture that the appropriate characterization is that the infinitesimal variation of $\mathbf{X}^{H}$ produced by $\mathbf{V}^{H}$,

$$
\delta \mathbf{X}^{H}=\mathcal{L}_{\mathbf{V}} \mathbf{X}^{H}=\left[\mathbf{V}^{H}, \mathbf{X}^{H}\right],
$$

is of the type $\{-$, phc $\}$, in order that the transformed vector field is again of the type (19). So, the characterization will read

$$
\left[\mathbf{V}^{H}, \mathbf{X}^{H}\right] \approx\{-, \text { phc }\} .
$$

Since Eq. (19) does not express the final form of the dynamics, we could produce more refined versions of (20). But, in the case of a Noether transformation, the invariance of the action is required not only on-shell but also off-shell, therefore the dynamics as given by (19) is the right one to be used.

Now let us prove that, when $\mathbf{V}^{H}$ generates a canonical transformation, relation (20) is exactly the characterization of a projectable Noether transformation. We can write $\mathbf{V}^{H}$ as

$$
\mathbf{V}^{H}=\left\{-, G^{H}\right\}
$$

for some function $G^{H}$, so that $\delta^{H} h=\left\{h, G^{H}\right\}$. To eliminate the weak equalities in (19), $\mathbf{X}^{H}$ can be written as

$$
\mathbf{X}^{H}=\frac{\partial}{\partial t}+\{-, H\}+\{-, \text { phc }\}+\phi_{\mu} \mathbf{Z}^{\mu}
$$

for some arbitrary vector fields $\mathbf{Z}^{\mu}$. Then, taking into account that

$$
\left[\mathbf{X}^{H}, \mathbf{V}^{H}\right] \approx\left\{-, \mathbf{X}^{H}\left(G^{H}\right)+\text { phc }\right\}-\mathbf{V}^{H}\left(\phi_{\mu}\right) \mathbf{Z}^{\mu},
$$

the requirement (20) becomes

$$
\mathbf{V}^{H}\left(\phi_{\mu}\right)=\text { phc }, \quad \mathbf{X}^{H}\left(G^{H}\right)=\text { phc }+f(t),
$$

where $f(t)$ is an unknown function of time. Notice that $G^{H}$ can be redefined by $G^{H} \rightarrow G^{H}$ $-\int f(t) d t$, since this does not change Eq. (21), and hence we have 


$$
\mathbf{V}^{H}\left(\phi_{\mu}\right)=\text { phc }, \quad \mathbf{X}^{H}\left(G^{H}\right)=\text { phc; }
$$

but since the functions $\lambda^{\mu}$ in the definition of $\mathbf{X}^{H}$ (18) are arbitrary, the second equation in (22) splits into

$$
\frac{\partial G^{H}}{\partial t}+\left\{G^{H}, H\right\}=\text { phc }
$$

and

$$
\left\{G^{H}, \phi_{\mu}\right\}=\text { phc. }
$$

Notice that (24) is just the first equation in (22).

It was proven in Ref. 16 that given a Noether transformation there exists a function $G^{H}$, whose pullback to velocity space is the standard conserved quantity $G^{L}$, satisfying these conditions (23) and (24); and conversely, that these conditions ensure that the transformation generated by $G^{H}$ through (17) and (16) is a Noether symmetry. What we have then obtained is a reformulation of (23) and (24) as commutativity conditions. To be more specific, we have proved the following result:

Theorem 1: An infinitesimal transformation in phase space is a canonical Noether transformation if and only if its vector field $\mathbf{V}^{H}$ satisfies

$$
\left[\mathbf{V}^{H}, \mathbf{X}^{H}\right] \approx\{-, \text { phc }\}, \quad \mathcal{L}_{\mathbf{V}^{H}} \Omega=0,
$$

where $\mathbf{X}^{H}$ is defined by (19) and $\Omega$ is the symplectic form in phase space.

(The contents of the second condition in (25) is that $\mathbf{V}^{H}$ generates canonical transformations.)

\section{B. Characterization using the evolution operator $\mathrm{K}$}

Now we will show an alternative characterization of Noether transformations in phase space that makes use of a special evolution operator that connects the phase space picture with the velocity space picture. Gauge systems derived from a variational principle exhibit evolution vector fields, either in the Lagrangian formulation or in the Hamiltonian one, that contain some arbitrariness, because of the gauge freedom. But one can also consider a third evolution operator that, unlike the previous ones, is fully deterministic. ${ }^{6}$ This is the operator $\mathbf{K}$ of Sec. II.

Using the operator $\mathbf{K}$, the Noether conditions (23) and (24) get the simpler form ${ }^{16}$

$$
\mathbf{K} \cdot G^{H}=0 .
$$

Our scope is to present these Noether conditions in a new form, combining Hamiltonian and Lagrangian transformations and involving commutations with both the pull-back operation and the evolution operator $\mathbf{K}$. This method has the advantage of its simplicity because, as we said, the operator $\mathbf{K}$ has none of the arbitrariness that plague the evolution vector fields in velocity space and phase space. In this sense, the commutation properties involving $\mathbf{K}$ will be the easiest ones to be used as a test of Noether symmetry. In order to do so, we will prepare some preliminary results.

First let us consider two infinitesimal transformations (leaving time invariant), $\delta^{H}$ in phase space, and $\delta^{L}$ in velocity space. In principle, they are unrelated, and do not necessarily describe symmetries. For a function $h(t, q, p)$ the variation is computed in terms of $\delta^{H} q$ and $\delta^{H} p$ as

$$
\delta^{H} h(t, q, p)=\frac{\partial h}{\partial q} \delta^{H} q+\frac{\partial h}{\partial p} \delta^{H} p,
$$

and similarly for a function $f(t, q, \dot{q})$ : 


$$
\delta^{L} f(t, q, \dot{q})=\frac{\partial f}{\partial q} \delta^{L} q+\frac{\partial f}{\partial \dot{q}} \delta^{L} \dot{q} .
$$

Using these relations, the definitions of $\mathcal{F} L$ and $\mathbf{K}$, and the chain rule, a straightforward computation shows that

$$
\begin{aligned}
\delta^{L} \mathcal{F} L^{*}(h)-\mathcal{F} L^{*}\left(\delta^{H} h\right)=\frac{\widehat{\partial h}}{\partial q}\left(\delta^{L} q-\widehat{\delta^{H} q}\right)+\frac{\widehat{\partial h}}{\partial p}\left(\delta^{L} \hat{p}-\widehat{\delta^{H} p}\right), \\
\delta^{L}(\mathbf{K} \cdot h)-\mathbf{K} \cdot \delta^{H} h=\left(\mathbf{K} \cdot \frac{\partial h}{\partial q}\right)\left(\delta^{L} q-\widehat{\delta^{H} q}\right)+\left(\mathbf{K} \cdot \frac{\partial h}{\partial p}\right)\left(\delta^{L} \hat{p}-\widehat{\delta^{H} p}\right)+\frac{\widehat{\partial h}}{\partial q}\left(\delta^{L} \dot{q}-\mathbf{K} \cdot \delta^{H} q\right) \\
+\frac{\partial h}{\partial p}\left(\delta^{L}(\mathbf{K} \cdot p)-\mathbf{K} \cdot \delta^{H} p\right),
\end{aligned}
$$

where we have written $\hat{h}$ for $\mathcal{F} L^{*}(h)$ to simplify the notation. As a consequence, we have:

Theorem 2: A necessary and sufficient condition in order that

$$
\delta^{L}(\mathbf{K} \cdot h)-\mathbf{K} \cdot \delta^{H} h=0
$$

for each function $h$, is that the transformations $\delta^{L}, \delta^{H}$ be related by

$$
\begin{gathered}
\delta^{L} q=\widehat{\delta^{H} q} \\
\delta^{L} \dot{q}=\mathbf{K} \cdot \delta^{H} q \\
\delta^{L} \hat{p}=\widehat{\delta^{H} p} \\
\delta^{L}(\mathbf{K} \cdot p)=\mathbf{K} \cdot \delta^{H} p .
\end{gathered}
$$

Moreover, then one also has $\delta^{L} \mathcal{F} L^{*}(h)-\mathcal{F} L^{*}\left(\delta^{H} h\right)=0$.

To prove the first assertion, one only has to take appropriate values for $h$ : taking $h=q^{i}$ or $h=p_{i}$ leads to the vanishing of the last two terms in (28); taking $h=\left(q^{i}\right)^{2} / 2$ leads to the vanishing of the first term; finally, taking $h=q^{i} p_{i}$ (not summed) does the rest.

In view of this, the last assertion is a direct consequence of (27).

From now on we suppose that the infinitesimal transformation in phase space is canonical, and let $G^{H}(t, q, p)$ a generating function for it (determined up to a function of time):

$$
\delta^{H} q=\left\{q, G^{H}\right\}=\frac{\partial G^{H}}{\partial p}, \quad \delta^{H} p=\left\{p, G^{H}\right\}=-\frac{\partial G^{H}}{\partial q} .
$$

We will need to know the partial derivatives of $\mathbf{K} \cdot h$. A direct calculation from the definition (6) yields

$$
\begin{gathered}
\frac{\partial(\mathbf{K} \cdot h)}{\partial q}=\mathbf{K} \cdot \frac{\partial h}{\partial q}+\frac{\partial^{2} L}{\partial q \partial q} \frac{\widehat{\partial h}}{\partial p}+\frac{\partial^{2} L}{\partial q \partial \dot{q}}\left(\mathbf{K} \cdot \frac{\partial h}{\partial p}\right), \\
\frac{\partial(\mathbf{K} \cdot h)}{\partial \dot{q}}=\frac{\widehat{\partial h}}{\partial q}+\frac{\partial^{2} L}{\partial \dot{q} \partial q} \frac{\widehat{\partial h}}{\partial p}+\frac{\partial^{2} L}{\partial \dot{q} \partial \dot{q}}\left(\mathbf{K} \cdot \frac{\partial h}{\partial p}\right) .
\end{gathered}
$$

These relations applied to $h=G^{H}$ yield 


$$
\begin{gathered}
\frac{\partial\left(\mathbf{K} \cdot G^{H}\right)}{\partial q}=-\mathbf{K} \cdot \delta^{H} p+\frac{\partial^{2} L}{\partial q \partial q} \widehat{\delta^{H} q}+\frac{\partial^{2} L}{\partial q \partial \dot{q}}\left(\mathbf{K} \cdot \delta^{H} q\right), \\
\frac{\partial\left(\mathbf{K} \cdot G^{H}\right)}{\partial \dot{q}}=-\widehat{\delta^{H} p}+\frac{\partial^{2} L}{\partial \dot{q} \partial q} \widehat{\delta^{H} q}+\frac{\partial^{2} L}{\partial \dot{q} \partial \dot{q}}\left(\mathbf{K} \cdot \delta^{H} q\right) .
\end{gathered}
$$

Now let us write $\delta^{L} f$ for $f=\mathbf{K} \cdot p=\partial L / \partial q$ and for $f=\hat{p}=\partial L / \partial \dot{q}$. We obtain the identities

$$
\begin{gathered}
0=\delta^{L}(\mathbf{K} \cdot p)-\frac{\partial^{2} L}{\partial q \partial q} \delta^{L} q-\frac{\partial^{2} L}{\partial q \partial \dot{q}} \delta^{L} \dot{q}, \\
0=\delta^{L} \hat{p}-\frac{\partial^{2} L}{\partial \dot{q} \partial q} \delta^{L} q-\frac{\partial^{2} L}{\partial \dot{q} \partial \dot{q}} \delta^{L} \dot{q} .
\end{gathered}
$$

Using these relations, Eqs. (33) and (34) become

$$
\begin{gathered}
\left.\frac{\partial\left(\mathbf{K} \cdot G^{H}\right)}{\partial q}=\delta^{L}(\mathbf{K} \cdot p)-\mathbf{K} \cdot \delta^{H} p+\frac{\partial^{2} L}{\partial q \partial q} \widehat{\delta^{H} q}-\delta^{L} q\right)+\frac{\partial^{2} L}{\partial q \partial \dot{q}}\left(\mathbf{K} \cdot \delta^{H} q-\delta^{L} \dot{q}\right), \\
\frac{\partial\left(\mathbf{K} \cdot G^{H}\right)}{\partial \dot{q}}=\delta^{L} \hat{p}-\widehat{\delta^{H} p}+\frac{\partial^{2} L}{\partial \dot{q} \partial q}\left(\widehat{\delta^{H} q}-\delta^{L} q\right)+\frac{\partial^{2} L}{\partial \dot{q} \partial \dot{q}}\left(\mathbf{K} \cdot \delta^{H} q-\delta^{L} \dot{q}\right) .
\end{gathered}
$$

So far we have not made any assumption on the relationship between $\delta^{H}$ and $\delta^{L}$, but from the preceding equations the following result is clear:

Theorem 3: Let $G^{H}(t, q, p)$ be the generator of an infinitesimal transformation $\delta^{H}$ in phase space (30). If we define an infinitesimal transformation $\delta^{L}$ in velocity space by

$$
\delta^{L} q:=\mathcal{F} L^{*}\left(\delta^{H} q\right), \quad \delta^{L} \dot{q}:=\mathbf{K} \cdot \delta^{H} q,
$$

then we have

$$
\begin{gathered}
\frac{\partial\left(\mathbf{K} \cdot G^{H}\right)}{\partial q}=\delta^{L} \mathbf{K} \cdot p-\mathbf{K} \cdot \delta^{H} p, \\
\frac{\partial\left(\mathbf{K} \cdot G^{H}\right)}{\partial \dot{q}}=\delta^{L} \mathcal{F} L^{*}(p)-\mathcal{F} L^{*}\left(\delta^{H} p\right) .
\end{gathered}
$$

Under the assumptions of the theorem, we can rewrite the commutation relations (27), (28) as

$$
\begin{gathered}
\delta^{L} \mathcal{F} L^{*}(h)-\mathcal{F} L^{*}\left(\delta^{H} h\right)=\frac{\widehat{\partial h}}{\partial p} \frac{\partial}{\partial \dot{q}}\left(\mathbf{K} \cdot G^{H}\right), \\
\delta^{L}(\mathbf{K} \cdot h)-\mathbf{K} \cdot \delta^{H} h=\left(\mathbf{K} \cdot \frac{\partial h}{\partial p}\right) \frac{\partial}{\partial \dot{q}}\left(\mathbf{K} \cdot G^{H}\right)+\frac{\widehat{\partial h}}{\partial p} \frac{\partial}{\partial q}\left(\mathbf{K} \cdot G^{H}\right) .
\end{gathered}
$$

The final step is to relate these relations with the condition (26), $\mathbf{K} \cdot G^{H}=0$, that characterizes the generators of projectable Noether transformations:

Theorem 4: Let $\delta^{H}$ be a canonical transformation in phase space, and let $\delta^{L}$ be defined as in (37). Then the following statements are equivalent:

(1) The commutation relation $\delta^{L}(\mathbf{K} \cdot h)-\mathbf{K} \cdot \delta^{H} h=0$ holds for each function $h(t, q, p)$;

(2) $\delta^{H}$ is a Noether transformation in phase space. 
To prove that the first condition implies the second one, notice that, using Theorems 2 and 3, if $G^{H}$ is a generator of $\delta^{H}, \mathbf{K} \cdot G^{H}$ is a function of time, $f(t)$. Redefinition of $G^{H}$ to $G^{H}-\int f(t)$ makes $\mathbf{K} \cdot G^{H}=0$, therefore, according to $(26), \delta^{H}$ is a Noether transformation in phase space. The converse is a direct consequence of (26) and Theorem 3.

Let us finally remark that we could have defined, instead of (37),

$$
\bar{\delta}^{L} q:=\mathcal{F} L^{*}\left(\delta^{H} q\right), \quad \bar{\delta}^{L} \dot{q}:=\frac{\mathrm{d}}{\mathrm{d} t} \bar{\delta}^{L} q .
$$

Here the Lagrangian transformation of $q$ is the pull-back of the Hamiltonian one, whereas the transformation of the velocity is the natural prolongation of the transformation of the position. This is the usual way to define the transformations of the velocities out of the transformations of the positions. Notice that, using Eq. (7),

$$
\bar{\delta}^{L} \dot{q}^{i}=\delta^{L} \dot{q}^{i}-[L]_{j} \mathcal{F} L^{*}\left(\frac{\partial \delta^{H} q^{i}}{\partial p_{j}}\right),
$$

so both transformations coincide when applied to solutions of the Euler-Lagrange equation (they coincide "on-shell"). With $\bar{\delta}^{L}$ instead of $\delta^{L}$ Eqs. (38) and (39) acquire an additional term that vanishes on-shell. Therefore $\delta^{L}$ as defined in Theorem 3 is more appropriate in order to give a neat characterization of a Noether transformation through commutation relations. Nevertheless, it is $\bar{\delta}^{L}$ that, when applied to the Lagrangian, gives a total derivative. Indeed, from (15), one has

$$
\bar{\delta}^{L} L=\frac{\mathrm{d}}{\mathrm{d} t} \mathcal{F} L^{*}\left(p \delta^{H} q-G^{H}\right) .
$$

\section{Characterization in velocity space}

To obtain a characterization in velocity space we first need to formulate the dynamics as a vector field in $\mathbf{R} \times \mathrm{T} Q$. The time evolution in a gauge theory is not unique until the gauge freedom has been removed-by way of some gauge fixing, for example. This is reflected in the ambiguities that are present in the Lagrangian time-evolution differential operator, which we recall from Sec. II:

$$
\mathbf{X}^{L}=\mathbf{X}_{0}+\eta^{\mu} \boldsymbol{\Gamma}_{\mu}, \quad \mathbf{X}_{0}=\frac{\partial}{\partial t}+\dot{q}^{i} \frac{\partial}{\partial q^{i}}+a^{i}(q, \dot{q}) \frac{\partial}{\partial \dot{q}^{i}} .
$$

Notice that projectable quantities have, according to (2), a well-defined unambiguous timederivative under this dynamics. The requirement of tangency of $\mathbf{X}^{L}$ to the primary Lagrangian constraint submanifold, defined by $\chi_{\mu} \approx 0$ (5), may lead to new constraints and to the determination of some of the functions $\eta^{\mu}$. At this point, new tangency requirements may occur. This is the Dirac's method in the Lagrangian formalism. ${ }^{6}$

Our aim is to give a tangent space characterization of a Noether transformation $\delta^{L} q(t ; q, \dot{q})$ that satisfies the property of being projectable to phase space, that is, $\delta^{L} q$ is the pullback of a canonical Noether transformation $\delta^{H} q, \delta^{L} q=\mathcal{F} L^{*}\left(\delta^{H} q\right)$. Notice at this point that we have two natural ways to define the dynamical time derivative $\delta^{L} \dot{q}$ in $\mathbf{R} \times \mathrm{T} Q$. Either by $\delta^{L} \dot{q}:=\mathbf{K} \cdot \delta^{H} q$ as in the preceding subsection, or by $\delta^{L} \dot{q}:=\mathbf{X}^{L} \cdot \delta^{L} q=\mathbf{X}_{0} \cdot \delta^{L} q$. According to (14), both definitions coincide only on the primary Lagrangian constraints submanifold. Consistency with the preceding subsection invites us to choose the definition $\delta^{L} \dot{q}:=\mathbf{K} \cdot \delta^{H} q$, and this is what we will do. So we take

$$
\mathbf{V}^{L}=\mathcal{F} L^{*}\left\{q, G^{H}\right\} \frac{\partial}{\partial q}+\mathbf{K} \cdot\left\{q, G^{H}\right\} \frac{\partial}{\partial \dot{q}} .
$$


We will use the results of the preceding subsection for Noether transformations, in particular

$$
\mathbf{K} \cdot \delta^{H} h=\delta^{L}(\mathbf{K} \cdot h)
$$

and its consequence

$$
\mathcal{F} L^{*}\left(\delta^{H} h\right)=\delta^{L} \mathcal{F} L^{*}(h),
$$

for any function $h$ on $\mathbf{R} \times \mathrm{T}^{*} Q$.

Notice from these relations that

$$
\mathbf{V}^{L} \cdot \mathcal{F} L^{*}(h)=\mathcal{F} L^{*}\left\{h, G^{H}\right\}:
$$

the action of $\mathbf{V}^{L}$ on a projectable function is a projectable function, that is, $\mathbf{V}^{L}$ is a projectable vector field-indeed it projects to $\mathbf{V}^{H}=\left\{-, G^{H}\right\}$.

Equation (44), and the fact that the primary Lagrangian constraints (plc) can be obtained as $\chi_{\mu}=\mathbf{K} \cdot \phi_{\mu}$, allow to compute

$$
\mathbf{V}^{L} \cdot \chi_{\mu}=\delta^{L} \chi_{\mu}=\delta^{L}\left(\mathbf{K} \cdot \phi_{\mu}\right)=\mathbf{K} \cdot\left(\delta^{H} \phi_{\mu}\right)=\mathbf{K} \cdot\left\{\phi_{\mu}, G^{H}\right\},
$$

but, according to (24),

$$
\left\{\phi_{\mu}, G^{H}\right\}=D_{\mu}^{\nu} \phi_{\nu}
$$

for some functions $D_{\mu}^{\nu}$. Therefore

$$
\mathbf{V}^{L} \cdot \chi_{\mu}=\delta^{L} \chi_{\mu}=\mathcal{F} L^{*}\left(D_{\mu}^{\nu}\right) \chi_{\nu}
$$

that is, $\mathbf{V}^{L}$ is tangent to the primary Lagrangian constraints surface $V_{1}, \mathbf{V}^{L}($ plc $)=$ plc.

Now let us use (14) and (44) to write

$$
\mathbf{V}^{L}\left(\mathbf{X}_{0} \cdot \mathcal{F} L^{*}(h)\right)+\mathbf{V}^{L}\left(\chi_{\mu} \frac{\partial v^{\mu}}{\partial \dot{q}} \mathcal{F} L^{*}\left(\frac{\partial h}{\partial p}\right)\right)=\mathbf{X}_{0} \cdot \mathcal{F} L^{*}\left(\delta^{H} h\right)+\chi_{\mu} \frac{\partial v^{\mu}}{\partial \dot{q}} \mathcal{F} L^{*}\left(\frac{\partial \delta^{H} h}{\partial p}\right) .
$$

The second piece in the right side is just a combination of plc, and so it is the second piece in the left side because of the tangency of $\mathbf{V}^{L}$ to the plc surface. Therefore

$$
\mathbf{V}^{L}\left(\mathbf{X}_{0} \cdot \mathcal{F} L^{*}(h)\right)-\mathbf{X}_{0} \cdot \mathcal{F} L^{*}\left(\delta^{H} h\right)=\text { plc, }
$$

or, using (45),

$$
\left[\mathbf{V}^{L}, \mathbf{X}_{0}\right]\left(\mathcal{F} L^{*} h\right)=\text { plc. }
$$

This result means that the commutator $\left[\mathbf{V}^{L}, \mathbf{X}_{0}\right]$ is, on the plc surface, a combination of the vector fields in the kernel of $\mathrm{T}(\mathcal{F} L)$, that is,

$$
\left[\mathbf{V}^{L}, \mathbf{X}_{0}\right]=\text { plc }+\alpha^{\mu} \boldsymbol{\Gamma}_{\mu},
$$

for some functions $\alpha^{\mu}$.

We need a second piece of information: the commutator $\left[\mathbf{V}^{L}, \boldsymbol{\Gamma}_{\mu}\right]$. Let us apply it to a configuration variable $q$. Since $\boldsymbol{\Gamma}_{\mu} \cdot q=0$ and $\boldsymbol{\Gamma}_{\mu} \cdot \delta^{L} q=\boldsymbol{\Gamma}_{\mu} \cdot \mathcal{F} L^{*}\left(\delta^{H} q\right)=0$, we get $\left[\mathbf{V}^{L}, \boldsymbol{\Gamma}_{\mu}\right] \cdot q$ $=0$. When applied to $\dot{q}$,

$$
\left[\mathbf{V}^{L}, \boldsymbol{\Gamma}_{\mu}\right] \cdot \dot{q}=\mathbf{V}^{L} \gamma_{\mu}-\boldsymbol{\Gamma}_{\mu} \mathbf{V}^{L}(\dot{q})=\mathbf{V}^{L} \gamma_{\mu}-\boldsymbol{\Gamma}_{\mu} \cdot\left(\mathbf{K} \cdot \delta^{H} q\right),
$$

where in the last step we have used the definition $\mathbf{V}^{L} \cdot \dot{q}=\mathbf{K} \cdot \delta^{H} q$. Taking into account the definition (3) and the property (11), we get 


$$
\begin{aligned}
{\left[\mathbf{V}^{L}, \boldsymbol{\Gamma}_{\mu}\right] \cdot \dot{q} } & =\mathbf{V}^{L}\left(\mathcal{F} L^{*}\left\{q, \phi_{\mu}\right\}\right)-\mathcal{F} L^{*}\left\{\delta^{H} q, \phi_{\mu}\right\} \\
& =\mathcal{F} L^{*}\left(\delta^{H}\left\{q, \phi_{\mu}\right\}-\left\{\delta^{H} q, \phi_{\mu}\right\}\right)=\mathcal{F} L^{*}\left\{q, \delta^{H} \phi_{\mu}\right\} .
\end{aligned}
$$

We can use again (46), $\delta^{H} \phi_{\mu}=\left\{\phi_{\mu}, G^{H}\right\}=D_{\mu}^{\nu} \phi_{\nu}$. Then,

$$
\left[\mathbf{V}^{L}, \boldsymbol{\Gamma}_{\mu}\right] \cdot \dot{q}=\mathcal{F} L^{*}\left\{q, D_{\mu}^{\nu} \phi_{\mu}\right\}=\left(\mathcal{F} L^{*} D_{\mu}^{\nu}\right) \gamma_{\nu},
$$

and therefore

$$
\left[\mathbf{V}^{L}, \boldsymbol{\Gamma}_{\mu}\right]=\left(\mathcal{F} L^{*} D_{\mu}^{\nu}\right) \boldsymbol{\Gamma}_{\nu},
$$

which agrees with the fact that $\mathbf{V}^{L}$ is projectable.

Putting together (48) and (51), we obtain that the vector field $\mathbf{V}^{L}$ satisfies

$$
\left[\mathbf{V}^{L}, \mathbf{X}^{L}\right]=\text { plc }+\beta^{\mu} \boldsymbol{\Gamma}_{\mu},
$$

for some functions $\beta^{\mu}(t ; q, \dot{q})$.

So we have proved the following result:

Theorem 5: Suppose that $G^{H}(t, q, p)$ generates a canonical Noether transformation, and let $\mathbf{V}^{L}$ be the vector field defined by it according to (43). Then $\mathbf{V}^{L}$ is a projectable vector field that projects to $\left\{-, G^{H}\right\}$, it is tangent to the primary Lagrangian constraint submanifold, and its commutation with the dynamics satisfies (52).

This result is analogous to that of Sec. III A. Here and there the commutator of the generator of the transformation with the evolution vector field gives as a result a term which is proportional to the arbitrary piece in the dynamics. Have we reached a necessary and sufficient condition for $\mathbf{V}^{L}$ to be a generator of a projectable Noether transformation? The answer in general is in the negative. Let us be more specific and consider a vector field $\mathbf{V}^{L}$, defined in (43), such that (a) it projects to $\left\{-, G^{H}\right\}$, (b) is tangent to the primary lagrangian constraint submanifold, and (c) satisfies (52). Then, using Eqs. (40) and (41) one arrives at

$$
\frac{\partial}{\partial \dot{q}}\left(\mathbf{K} \cdot G^{H}\right)=0, \quad \frac{\partial}{\partial q}\left(\mathbf{K} \cdot G^{H}\right)=\text { plc },
$$

whereas the right conditions for $\left\{-, G^{H}\right\}$ to generate a Noether transformation in phase spacewhich implies that $\mathbf{V}^{L}$ generates a Noether transformation in tangent space-are, according to the discussion in the preceding section,

$$
\frac{\partial}{\partial \dot{q}}\left(\mathbf{K} \cdot G^{H}\right)=0, \quad \frac{\partial}{\partial q}\left(\mathbf{K} \cdot G^{H}\right)=0,
$$

which is more restrictive than (53). However, in most cases of interest, the plc do not restrict the configuration variables alone, and then (53) and (54) are equivalent. This is the case indeed in many physical applications of gauge systems, as in string theory, Yang-Mills theory, or general relativity. In such cases we have arrived at a characterization of the vector field $\mathbf{V}^{L}$ for it to generate a Noether transformation.

The case where the plc do restrict the configuration variables is rather unusual, and it might be considered as an unfortunate choice of the configuration space-some comments on this issue can be found in Ref. 8. The second example in the following section, though formal and with no physical interest, exhibits this feature; in this case, conditions (53) are not sufficient for $\mathbf{V}^{L}$ to generate a Noether transformation.

Let us finally recall that the action of the vector field $\mathbf{V}^{L}$, associated to a projectable Noether transformation, on the Lagrangian $L$ does not give in general a total derivative. The transformation that indeed gives a total derivative is $\bar{\delta}^{L}$ - see the end of Sec. III B. 


\section{The algebra of projectable Noether symmetries}

Consider a canonical Noether symmetry generated by $G^{H}$. The projectability of (43),

$$
\mathbf{V}^{L}=\mathcal{F} L^{*}\left\{q, G^{H}\right\} \frac{\partial}{\partial q}+\mathbf{K} \cdot\left\{q, G^{H}\right\} \frac{\partial}{\partial \dot{q}},
$$

to the canonical generator of Noether symmetries,

$$
\mathbf{V}^{H}=\left\{-, G^{H}\right\},
$$

allows to obtain some results concerning the algebra of the vector fields associated to projectable Noether symmetries. If $\mathbf{V}_{1}^{L}$ and $\mathbf{V}_{2}^{L}$ are two such vector fields associated with the canonical generating functions $G_{1}^{H}$ and $G_{2}^{H}$, then it is straightforward to show that the commutator $\left[\mathbf{V}_{1}^{L}, \mathbf{V}_{2}^{L}\right]$ projects to $\left\{-,\left\{G_{2}^{H}, G_{1}^{H}\right\}\right\}$, that is,

$$
\left[\mathbf{V}_{1}^{L}, \mathbf{V}_{2}^{L}\right] \cdot \mathcal{F} L^{*}(h)=\left\{h,\left\{G_{2}^{H}, G_{1}^{H}\right\}\right\} .
$$

In the particular case that the set of independent canonical generators $G_{i}$ span a Lie algebra,

$$
\left\{G_{i}^{H}, G_{j}^{H}\right\}=C_{i j}^{k} G_{k}^{H},
$$

with $C_{i j}^{k}$ constants, then their associated vector fields $\mathbf{V}_{i}^{L}$ in tangent space satisfy the same Lie algebra structure,

$$
\left[\mathbf{V}_{i}^{L}, \mathbf{V}_{j}^{L}\right]=C_{i j}^{k} \mathbf{V}_{k}^{L} .
$$

In the case that the quantities in (55) are not constants but functions of the variables (this is the case of a "soft" algebra generating a "quasigroup"), ${ }^{23}$ this last equality does not hold, but we still have the opportunity to get - up to pieces linear in the primary constraints-the structure functions in phase space by Lagrangian methods. This goes as follows. Consider (55) for some functions $C_{i j}^{k}$. Consider also the pull-back to tangent space of the canonical generating functions, $G_{i}^{L}=\mathcal{F} L^{*}\left(G_{i}^{H}\right)$. Then

$$
\mathbf{V}_{j}^{L} \cdot G_{i}^{L}=\mathbf{V}_{j}^{L} \cdot \mathcal{F} L^{*}\left(G_{i}^{H}\right)=\mathcal{F} L^{*}\left(\delta_{j}^{H} G_{i}^{H}\right)=\mathcal{F} L^{*}\left\{G_{i}^{H}, G_{j}^{H}\right\}=\mathcal{F} L^{*}\left(C_{i j}^{k} G_{k}^{H}\right)=\mathcal{F} L^{*}\left(C_{i j}^{k}\right) G_{k}^{L} .
$$

That is, we can retrieve - up to primary constraints - the structure functions of the canonical gauge generators by simply computing the variations under the vector fields $\mathbf{V}^{L}$ of the Noether conserved quantities in tangent space. This method has been implicitly used in a series of papers ${ }^{24-26}$ that analyze the relationship between the Lagrangian and Hamiltonian descriptions of the gauge group structure for generally covariant theories.

\section{SOME EXAMPLES}

Example 1: Let us consider the Lagrangian

$$
L=\frac{1}{2} e^{-\omega} \dot{\mathbf{x}}^{2}+\frac{1}{2} e^{\omega} m^{2},
$$

which describes a free particle in Minkowski's space-time. A standard analysis yields the momenta $(\mathbf{p}, \pi)$ of the variables $(\mathbf{x}, \omega)$, a Hamiltonian function, and a primary Hamiltonian constraint:

$$
\hat{\mathbf{p}}=e^{-\omega} \dot{\mathbf{x}}, \quad \hat{\pi}=0, \quad H=\frac{1}{2} e^{\omega}\left(\mathbf{p}^{2}-m^{2}\right), \quad \phi^{0}=\pi .
$$


The stabilization algorithm yields a secondary Hamiltonian constraint

$$
\phi^{1}=\left\{\phi^{0}, H\right\}=-H .
$$

The evolution operator $\mathbf{K}$ is given by

$$
\mathbf{K} \cdot h=\mathcal{F} L^{*} \frac{\partial h}{\partial t}+\dot{\mathbf{x}} \mathcal{F} L^{*} \frac{\partial h}{\partial \mathbf{x}}+\dot{\omega} \mathcal{F} L^{*} \frac{\partial h}{\partial \omega}+\chi \mathcal{F} L^{*} \frac{\partial h}{\partial \pi},
$$

where we have denoted by $\chi$ the primary Lagrangian constraint

$$
\chi:=\mathbf{K} \cdot \phi^{0}=\frac{1}{2}\left(e^{\omega} m^{2}-e^{-\omega} \dot{\mathbf{x}}^{2}\right)
$$

It is clear that the projectable functions are those not depending on $\dot{\omega}$, and indeed the kernel of $\mathrm{T}(\mathcal{F} L)$ is spanned by

$$
\boldsymbol{\Gamma}=\frac{\partial}{\partial \dot{\omega}}
$$

Notice therefore that $\chi$ is a projectable constraint, and so

$$
\chi=\mathcal{F} L^{*}\left(\phi^{1}\right),
$$

whereas

$$
\mathbf{K} \cdot \phi^{1}=\dot{\omega} \chi
$$

which is not a new constraint. Finally, we give the Euler-Lagrange equations

$$
[L]_{\mathbf{x}}=e^{-\omega}(\dot{\omega} \dot{\mathbf{x}}-\ddot{\mathbf{x}}), \quad[L]_{\omega}=\chi .
$$

At the first stage of the stabilization algorithm the Hamiltonian evolution operator is

$$
\mathbf{X}^{H}=\frac{\partial}{\partial t}+e^{\omega} \mathbf{p} \frac{\partial}{\partial \mathbf{x}}-\frac{1}{2} e^{\omega}\left(\mathbf{p}^{2}-m^{2}\right) \frac{\partial}{\partial \pi}+\lambda \frac{\partial}{\partial \omega}+\pi \mathbf{Z}
$$

where the function $\lambda$ and the vector field $\mathbf{Z}$ are arbitrary.

Now let us study the gauge transformations. From the general theory, a gauge generator has the form $G^{H}=\dot{\varepsilon} G_{0}+\varepsilon G_{1}$, where $\varepsilon$ is an arbitrary function of time and the functions $G_{i}$ are determined such that $\mathbf{K} \cdot G^{H}=0 .{ }^{10} G_{0}$ is a first-class primary Hamiltonian constraint, which in this example turns out to be $e^{-\omega} \pi$. The result is

$$
G^{H}=e^{-\omega}\left(\dot{\varepsilon} \phi^{0}-\varepsilon \phi^{1}\right) .
$$

Its associated infinitesimal transformation is given by the vector field

$$
\mathbf{V}^{H}=\varepsilon \mathbf{p} \frac{\partial}{\partial \mathbf{x}}+\dot{\varepsilon} e^{-\omega} \frac{\partial}{\partial \omega}+\dot{\varepsilon} e^{-\omega} \pi \frac{\partial}{\partial \pi}
$$

Let us check the quasiinvariance of $\mathbf{X}^{H}$ :

$$
\left[\mathbf{V}^{H}, \mathbf{X}^{H}\right]=\left(e^{-\omega}(-\ddot{\varepsilon}+\lambda \dot{\varepsilon})+\mathbf{V}^{H} \cdot \lambda\right) \frac{\partial}{\partial \omega}+\pi\left(e^{-\omega}(-\ddot{\varepsilon}+\lambda \dot{\varepsilon}) \frac{\partial}{\partial \pi}+\left[\mathbf{V}^{H}, \mathbf{Z}\right]+e^{-\omega} \dot{\varepsilon} \mathbf{Z}\right)
$$

which is weakly $\{-$, phc $\}$. 
The vector field $\mathbf{V}^{L}$ of (43) is

$$
\mathbf{V}^{L}=e^{-\omega}\left(\varepsilon \mathbf{x} \frac{\partial}{\partial \mathbf{x}}+\dot{\varepsilon} \frac{\partial}{\partial \omega}+\dot{\varepsilon} \dot{\mathbf{x}} \frac{\partial}{\partial \dot{\mathbf{x}}}+\left(\dot{\varepsilon} \dot{\omega}-\ddot{\varepsilon} e^{-\omega}\right) \frac{\partial}{\partial \dot{\omega}}\right)
$$

A direct computation then shows that, as differential operators,

$$
\mathbf{V}^{L} \circ \mathbf{K}-\mathbf{K} \circ \mathbf{V}^{H}=0 .
$$

Finally let us consider the Lagrangian dynamical vector field,

$$
\mathbf{X}^{L}=\frac{\partial}{\partial t}+\dot{\mathbf{x}} \frac{\partial}{\partial \mathbf{x}}+\dot{\omega} \frac{\partial}{\partial \omega}+\dot{\omega} \dot{\mathbf{x}} \frac{\partial}{\partial \dot{\mathbf{x}}}+\eta \frac{\partial}{\partial \dot{\omega}},
$$

where $\eta$ is an arbitrary function. Then we obtain

$$
\left[\mathbf{V}^{L}, \mathbf{X}^{L}\right]=e^{-\omega}\left(-\dddot{\varepsilon}+2 \ddot{\varepsilon} \dot{\omega}-\dot{\varepsilon} \dot{\omega}^{2}+\dot{\varepsilon} e^{-\omega} \eta+\mathbf{V}^{L} \cdot \eta\right) \frac{\partial}{\partial \dot{\omega}},
$$

which is proportional to $\boldsymbol{\Gamma}$.

Moreover, bearing in mind the remarks at the end of Sec. III B, we can define the Lagrangian transformation of the velocities as the time-derivatives of the transformation of the positions, thus obtaining a slightly different vector field $\overline{\mathbf{V}}^{L}$; indeed,

$$
\overline{\mathbf{V}}^{L}=\mathbf{V}^{L}-\varepsilon[L]_{\mathbf{x}} \frac{\partial}{\partial \dot{\mathbf{x}}}
$$

Then the Noether condition can be checked for this transformation:

$$
\overline{\mathbf{V}}^{L} \cdot L=\frac{\mathrm{d}}{\mathrm{d} t}\left(\varepsilon e^{-\omega} L\right)
$$

Example 2: Here we show that, in general, the conditions stated by Theorem 5 are not sufficient for $\mathbf{V}^{L}$ to define a Noether transformation. Let us consider

$$
L=\frac{1}{2} \dot{x}^{2}-\frac{1}{2} y^{2}
$$

The momenta $\left(p_{x}, p_{y}\right)$ of the variables $(x, y)$, a Hamiltonian function and a primary Hamiltonian constraint are

$$
\hat{p}_{x}=\dot{x}, \quad \hat{p}_{y}=0, \quad H=\frac{1}{2} p_{x}^{2}+\frac{1}{2} y^{2}, \quad \phi^{0}=p_{y} .
$$

The stabilization algorithm yields a secondary Hamiltonian constraint

$$
\phi^{1}=\left\{\phi^{0}, H\right\}=-y \text {. }
$$

The evolution operator $\mathbf{K}$ is given by

$$
\mathbf{K} \cdot h=\mathcal{F} L * \frac{\partial h}{\partial t}+\dot{x} \mathcal{F} L * \frac{\partial h}{\partial x}+\dot{y} \mathcal{F} L * \frac{\partial h}{\partial y}-y \mathcal{F} L * \frac{\partial h}{\partial p_{y}} \text {. }
$$

Notice that there are a primary Lagrangian constraint and a secondary one, 


$$
\chi^{1}=-y, \quad \chi^{2}=-\dot{y} .
$$
by

The projectable functions are those not depending on $\dot{y}$, and the kernel of $\mathrm{T}(\mathcal{F} L)$ is spanned

$$
\boldsymbol{\Gamma}=\frac{\partial}{\partial \dot{y}} .
$$

The Euler-Lagrange equations are

$$
[L]_{x}=-\ddot{x}, \quad[L]_{y}=-y,
$$

and so the Lagrangian evolution operator may be taken as

$$
\mathbf{X}_{0}^{L}=\dot{x} \frac{\partial}{\partial x}+\dot{y} \frac{\partial}{\partial y} .
$$

Let us consider the function

$$
G^{H}=p_{y} y,
$$

whose associated infinitesimal transformation is the vector field in phase space

$$
\mathbf{V}^{H}=y \frac{\partial}{\partial y}-p_{y} \frac{\partial}{\partial p_{y}},
$$

and defines the vector field in tangent space

$$
\mathbf{V}^{L}=y \frac{\partial}{\partial y}+\dot{y} \frac{\partial}{\partial \dot{y}} .
$$

It is easily checked that $\mathbf{V}^{L}$ projects to $\mathbf{V}^{H}$. It is clear that $\mathbf{V}^{L} \cdot \chi^{1}=\chi^{1}$, and so it is tangent to the primary Lagrangian constraint submanifold. And also we have $\left[\mathbf{V}^{L}, \mathbf{X}_{0}^{L}\right]=0$.

In spite of satisfying these three conditions of Theorem $5, \mathbf{V}^{L}$ is not a projectable Noether transformation. We can see this in several ways. On the one hand, $\mathbf{K} \cdot G^{H}=-y^{2}$, which is not zero (notice, however, that since this is a primary Lagrangian constraint then $G^{H}$ corresponds to a nonprojectable Noether transformation, see Refs. 13 and 15). On the other hand, we can compute

$$
\left(\mathbf{V}^{L} \circ \mathbf{K}-\mathbf{K} \circ \mathbf{V}^{H}\right) \cdot h=-2 y \frac{\widehat{\partial h}}{\partial p_{y}},
$$

which is not zero. Finally, using the transformation $\overline{\mathbf{V}}^{L}$ as before, we have

$$
\overline{\mathbf{V}}^{L} \cdot L=-y^{2}
$$

which is not a total derivative.

Finally, we use this example to illustrate item (b) in the list of properties of gauge theories given in the introduction. Take the conserved quantity $G^{H}=p_{x}+p_{y} y$ in phase space. It generates, through Poisson bracket, an infinitesimal symmetry transformation $\delta^{H}$ whose pull-back to velocity space is $\delta^{L} x=1, \delta^{L} y=y$; this gives $\delta^{L} L=-y^{2}$, which is not a total derivative and thus $\delta^{L}$ is not a Noether symmetry. 


\section{CONCLUSIONS}

In this paper we have introduced some characterizations of Noether symmetries based upon some specific properties of commutativity with the dynamics. This presentation entails a shift of focus with respect to the standard introductions to Noether symmetries.

To our knowledge, the only characterization of Noether symmetries in gauge theories, not relying on properties of the conserved quantity, is the invariance of the action under these transformations. Our contribution is a new characterization of such symmetries which is set up in the realm of dynamics, either Lagrangian or Hamiltonian. This study concerns those Noether symmetries that are projectable to phase space (what we call canonical Noether transformations).

For canonical Noether symmetries we obtain a characterization in phase space that clearly generalizes the results that hold for regular (not gauge) theories. We also provide an alternative characterization by using the unambiguous evolution operator that connects the formulations in phase space and in tangent space; this new characterization is very appropriate because of its simplicity, since it is set up with the only use of the Lagrangian function and its partial derivatives. Finally, we give a characterization in velocity space applicable to most dynamical theories with physical contents.

In summary, we give an answer to the question of extending the property of commutation of the Noether symmetry with the dynamics, as expressed by Eq. (1), to singular Lagrangians. This answer is presented as three characterizations that may serve as a useful test of Noether symmetry for gauge theories with reference neither to the action nor to the conserved quantity.

\section{ACKNOWLEDGMENTS}

X.G. acknowledges financial support by CICYT Project TAP 97-0969-C03. J.M.P. acknowledges financial support by CICYT, AEN98-0431, and CIRIT, GC 1998SGR.

\footnotetext{
${ }^{1}$ P. J. Olver, Applications of Lie Groups to Differential Equations, 2nd ed. (Springer, New York, 1993).

${ }^{2}$ R. Abraham and J. E. Marsden, Foundations of Mechanics, 2nd ed. (Addison-Wesley, Reading, 1978).

${ }^{3}$ V. I. Arnol'd, Mathematical Methods of Classical Mechanics, 2nd ed. (Springer-Verlag, New York, 1989).

${ }^{4}$ P. A. M. Dirac, "Generalized Hamiltonian dynamics," Can. J. Math. 2, 129-148 (1950).

${ }^{5}$ P. A. M. Dirac, Lectures on Quantum Mechanics (Yeshiva University Press, New York, 1964).

${ }^{6}$ C. Batlle, J. Gomis, J. M. Pons, and N. Román, "Equivalence between the Lagrangian and Hamiltonian formalisms for constrained systems," J. Math. Phys. 27, 2953-2962 (1986).

${ }^{7}$ J. F. Cariñena, “Theory of singular Lagrangians," Fortschr. Phys. 38, 641-679 (1990).

${ }^{8}$ G. Marmo, N. Mukunda, and J. Samuel, "Dynamics and symmetry for constrained systems: a geometrical analysis," Riv. Nuovo Cimento 6, 1-62 (1983).

${ }^{9}$ A. Hanson, T. Regge, and C. Teitelboim, Constrained Hamiltonian Systems, (Accademia Nazionale dei Lincei, Roma, 1976).

${ }^{10}$ X. Gràcia and J. M. Pons, "Gauge generators, Dirac's conjecture, and degrees of freedom for constrained systems," Ann. Phys. (N.Y.) 187, 355-368 (1988)

${ }^{11} \mathrm{X}$. Gràcia and J. M. Pons, "Noether transformations with vanishing conserved quantity," Ann. Inst. Henri Poincaré, Sect. A 61, 315-327 (1994).

${ }^{12}$ K. Kamimura, "Singular Lagrangian and constrained Hamiltonian systems, generalized canonical formalism," Nuovo Cimento Soc. Ital. Fis., B 68, 33-54 (1982).

${ }^{13}$ X. Gràcia and J. M. Pons, "A Hamiltonian approach to Lagrangian Noether transformations," J. Phys. A 25, 6357-6369 (1992).

${ }^{14}$ X. Gràcia and J. Roca, "Covariant and noncovariant gauge transformations for the conformal particle," Mod. Phys. Lett. A 8, 1747-1761 (1993).

${ }^{15}$ J. A. García and J. M. Pons, "Rigid and gauge Noether symmetries for constrained systems," Int. J. Mod. Phys. A (to be published), hep-th/9908151.

${ }^{16}$ C. Batlle, J. Gomis, X. Gràcia, and J. M. Pons, "Noether's theorem and gauge transformations: application to the bosonic string and $C P_{2}^{n-1}$ model,"' J. Math. Phys. 30, 1345-1350 (1989).

${ }^{17}$ X. Gràcia and J. M. Pons, "On an evolution operator connecting Lagrangian and Hamiltonian formalisms," Lett. Math. Phys. 17, 175-180 (1989).

${ }^{18}$ J. M. Pons, "New relations between Hamiltonian and Lagrangian constraints," J. Phys. A 21, 2705-2715 (1988).

${ }^{19}$ X. Gràcia, "Fibre derivatives: some applications to singular lagrangians,"' Rep. Math. Phys. 45, 67-84 (2000).

${ }^{20} \mathrm{X}$. Gràcia and J. M. Pons, "Singular lagrangians: Some geometric structures along the Legendre's map"' (submitted).

${ }^{21}$ M. J. Gotay, J. M. Nester, and G. Hinds, "Presymplectic manifolds and the Dirac-Bergmann theory of constraints," J. Math. Phys. 19, 2388-2399 (1978).
} 
${ }^{22}$ M. J. Bergvelt and E. A. de Kerf, “The Hamiltonian structure of Yang-Mills theories and instantons I," Physica A 139, 101-124 (1986).

${ }^{23}$ I. A. Batalin, "Quasigroup construction and first class constraints," J. Math. Phys. 22, 1837-1850 (1981).

${ }^{24}$ J. M. Pons, D. C. Salisbury, and L. C. Shepley, "Gauge transformations in the Lagrangian and Hamiltonian formalisms of generally covariant systems," Phys. Rev. D 55, 658-668 (1997), gr-qc/9612037.

${ }^{25}$ J. M. Pons, D. C. Salisbury, and L. C. Shepley, "Gauge transformations in Einstein-Yang-Mills theories," J. Math. Phys. 41, 5557-5571 (2000), gr-qc/9912086.

${ }^{26}$ J. M. Pons, D. C. Salisbury, and L. C. Shepley, "'Gauge group and reality conditions in Ashtekar's complex formulation of canonical gravity," Phys. Rev. D (to be published), gr-qc/9912085. 